\title{
Aspect de la construction navale à Sulawesi (1969-1999)
}

Aspects of naval construction in Sulawesi (1969-1999)

Aspecto de la construcción naval de Sulawesi (1969-1999)

Jean-Claude Amos

\section{CpenEdition}

\section{Journals}

Édition électronique

URL : https://journals.openedition.org/tc/283

DOI : $10.4000 /$ tc. 283

ISSN : 1952-420X

\section{Éditeur}

Éditions de l'EHESS

\section{Édition imprimée}

Date de publication : 1 janvier 2001

Pagination : 121-139

ISSN : 0248-6016

Référence électronique

Jean-Claude Amos, « Aspect de la construction navale à Sulawesi (1969-1999)», Techniques \& Culture [En ligne], 35-36 | 2001, mis en ligne le 11 septembre 2012, consulté le 29 septembre 2022. URL :

http://journals.openedition.org/tc/283 ; DOI : https://doi.org/10.4000/tc.283

Ce document a été généré automatiquement le 29 septembre 2022

Tous droits réservés 


\title{
Aspect de la construction navale à Sulawesi (1969-1999)
}

\author{
Aspects of naval construction in Sulawesi (1969-1999) \\ Aspecto de la construcción naval de Sulawesi (1969-1999)
}

\author{
Jean-Claude Amos
}

1 Ce domaine est d'accès difficile. Problème du truchement, en premier lieu. Non que les informateurs ne maîtrisent la bahasa indonesia -le malais, dont elle est un avatar modernisé et romanisé, étant depuis longtemps la lingua franca de l'archipel-, ni même parce que le vocabulaire du métier change d'un lieu à un autre. Mais il y a surtout que les réponses à des questions précises varient... à quelques heures ou mètres de distance. Ne pas répondre serait inamical, voire une perte de face; autant raconter n'importe quoi. Notons, pour ne pas avoir à multiplier plus loin les précautions oratoires, que tout ce qui ne relève pas de l'observation directe, dans cet article ou ses sources, doit être accueilli avec prudence.

2 Problème, également, de l'immensité du sujet: il serait difficile de limiter son champ d'observation sans avoir une vue générale de l'ancienneté et du foisonnement de la marine indonésienne.

3 Enfin, rareté des sources. Il est surprenant que les bateaux de l'Insulinde n'aient pas été l'objet d'une étude systématique, comme ceux du Pacifique ou de la Chine. Les ouvrages de Pâris (1843) et Hornell (1936) n'y touchent que partiellement et tout le teste se réduit à de minces volumes épuisés et des articles de revues, inaccessibles au commun des mortels (Collins 1937; Dick 1975; Liebner 1993; Horridge 1979). Doit-on s'en étonner, sachant que la majeure partie des études concernant les bateaux de travail des côtes de France date du dernier quart de notre siècle ? Que n'a-t-on sauvé deux ou trois coques de voiliers de charge indonésiens, dont certains héritaient d'une tradition multiséculaire, comme on l'a fait de plusieurs pirogues au Musée de la mer de Jakarta! Ici un bateau abandonné sut une plage disparaît en moins d'un an. 


\section{Les chantiers navals}

Les chantiers de Marumasa et de Tana Beru, à l'extrême sud de Sulawesi, sont les plus importants du monde dans leur genre. Chacun, sur plus de deux kilomètres de plage, aligne un fouillis dense de coques en construction, du perahu de pêche de dix mètres au pinisi $^{2}$ ventru de deux cent tonnes, et donne de l'ouvrage à près de quinze cent chefs de famille (photo 1).

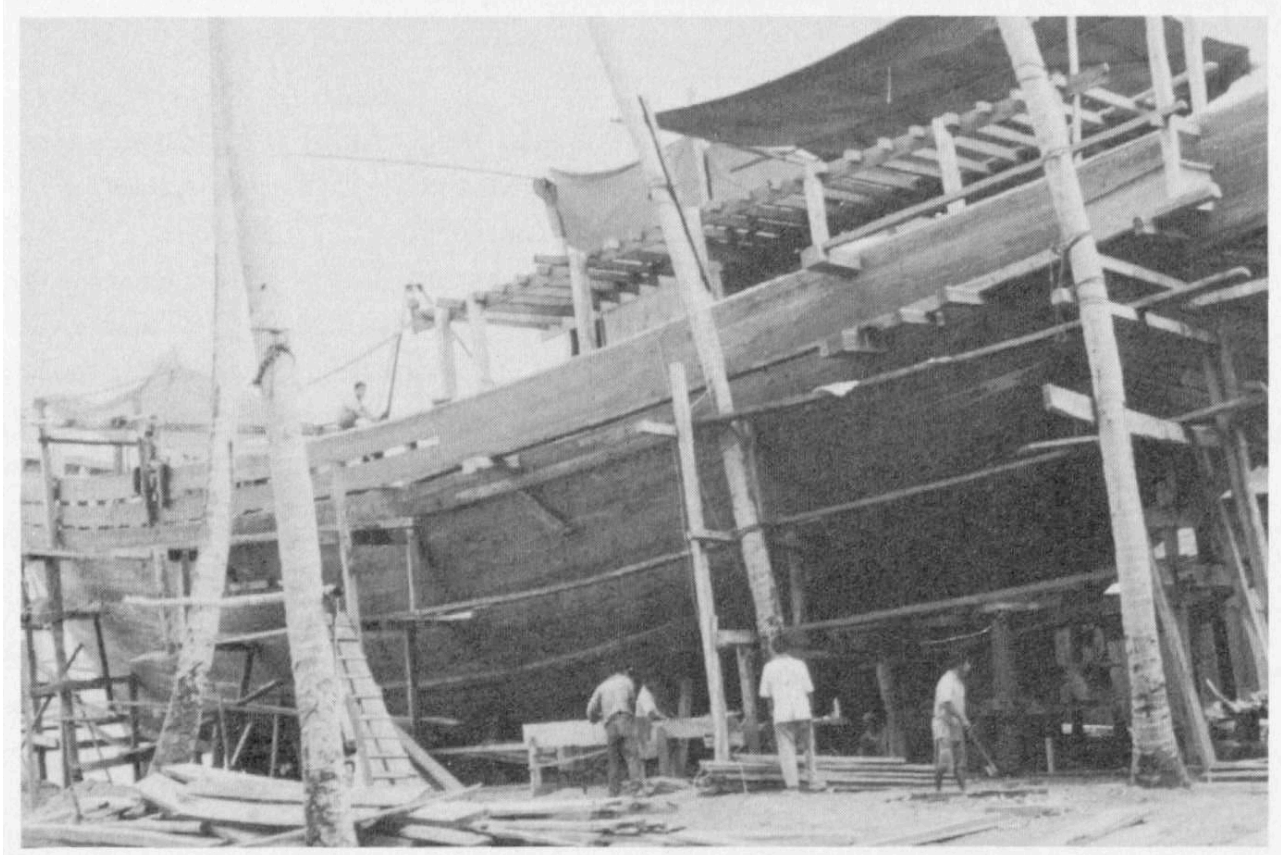

Photo 1. Construction d'un cargo pinisi en bois, Sulawesi (cl. J.-Cl. Amos)

5 Mais cette activité est entrée dans une période critique. À Tana Beru, on signale, comme de mauvais augure, le départ récent de 300 compagnons, partis chercher du travail jusqu'en Malaisie. Les patrons se plaignent de la raréfaction des belles bordailles, et surtout, du prix du bois, « excessivement haut ». Doléance sempiternelle, certes, mais corroborée par des articles de la presse nationale : les immenses forêts de Kalimantan, principal fournisseur en bois d'œuvre de l'archipel, auraient été trop et mal exploitées sur leurs lisières maritimes, les billes devant être maintenant cherchées en des lieux à peine accessibles.

Les armateurs sont, pour la plupart, des citoyens indonésiens, appartenant souvent à la communauté chinoise ; ils commandent principalement des barques de petit tonnage pour le cabotage et la pêche, extrêmement actifs dans un pays qui compte plus de cinq milliers d'îles habitées au sein d'une zone maritime couvrant trois fois la superficie de la Méditerranée.

7 Cependant, une quinzaine de gros entrepreneurs, à Tana Beru -et probablement autant à Maramusa- construisent toujours des pinisi de vingt à trente mètres, désormais motorisés et ne conservant qu'un gréement résiduel. Y sont lancés chaque année cinq à dix de ces « cargos mixtes » en bois, dont la construction nécessite, selon la taille, entre dix et douze mois de travail. Avec une espérance de vie limitée à dix ou quinze ans; mais on prétend qu'un armateur habile n'a besoin que d'une seule année pour amortir son investissement. 
8 Le prix moyen d'un pinisi varie énormément... selon les informateurs. On pourrait, en moyenne, tabler sur $60000 \$$ US. Cela paraît peu, mais pour un citoyen de base indonésien, c'est un chiffre astronomique.

On signale, avec un espoir non dissimulé, l'arrivée de nouveaux commanditaires d'Amérique du Nord et du Japon, probablement entrepreneurs de charters, voire amateurs de vieille marine. Ainsi construit-on actuellement à Tana Beru une « réplique » d'un vaisseau de la V.O.C. (Compagnie hollandaise des Indes Orientales).

10 L'impression générale n'est pas celle d'une activité fébrile. Sur la plupart des barques travaillent avec circonspection deux ou trois charpentiers, voire un seul. Un pinisi mobilise une équipe d'une douzaine d'hommes, soit le maître de hache ${ }^{3}$, omnipotent, un peu plus de la moitié de compagnons (dits sawi-sawi, "fourmis») et quelques apprentis, qui changeront de statut après avoir fait leurs preuves. Ce sont souvent des proches du maître ; les liens de parenté sont censés favoriser l'harmonie. On ne voit pas de femmes. Elles ne doivent pas, d'ailleurs, grimper sur une coque avant le pontage. Superstition qui n'étonnerait pas un vieux bat-la-houle du Ponant.

11 Les compagnons sont des Bugis, mais appartiennent à une sorte de caste professionnelle. Il serait très mal vu de recruter à l'extérieur! Si bien que tous, fils et petits-fils de charpentiers de marine, ont plus ou moins un lien de cousinage. Ils parlent le konjo, un dialecte restreint à la région des chantiers.

12 Leur savoir-faire est proverbial. Ils ont essaimé dans l'archipel et créé d'autres chantiers, en particulier à Kalimantan. Cela relève d'ailleurs d'une vieille tradition des Bugis, qui organisèrent au XVIII ${ }^{e}$ siècle, supplantant les Macassars, une confédération maritime, et fondèrent des colonies, notamment de part et d'autre du détroit de Malacca (Lineton 1975 ; Pelras 1975). On lit quelquefois que les charpentiers seraient de purs terriens, mais le contraire nous a été affirmé sur place: nombre d'entre eux partiraient chaque année pour quelque périple, surtout en octobre et novembre, peutêtre la saison creuse des chantiers.

13 On dit aussi qu'ils seraient tenus à part de la communauté paysanne bugis. Cet aspect des choses n'est pas de notre compétence. Mais nous nous souvenons de la séparation, matérialisée par la rivière, entre pêcheurs et riziculteurs dans le village soundanais où nous avions séjourné quelques mois en 1968. Nos hôtes, petits paysans, nous avaient mis en garde contre la fréquentation des gens de mer.

\section{Techniques actuelles}

14 Sauf depuis l'introduction récente de perceuses et de rabots électriques de bricoleur, tout se fait avec un outillage réduit : hache, herminette, tarière, égoïne, ciseau, maillet. À Tana Beru fonctionne une scie circulaire sur plateau; on a apparemment abandonné le sciage en long manuel, naguère de règle dans les chantiers asiatiques. Décrire le processus de construction demanderait un fort cahier. On ne s'attachera ici qu'à quelques points, souvent mal interprétés.

15 Comme partout en Extrême-Orient, on construit «bordé d'abord ». Il n'y a ni plans, ni gabarits, ni «bâton de joseph »... Seul compte l'œil du maître de hache. Ce qui peut expliquer que l'on ne trouve jamais deux unités identiques. 

second d'un can à l'autre, à intervalles rigoureusement semblables. Or, l'opération est réalisée avec de médiocres tarières adaptées à des perceuses de petite capacité : respecter la perpendicularité prouve un bon tour de main. On engage de fines gournables dans le nouveau bordage, en faisant dépasser de quelques centimètres les deux premières, enfoncées dans les trous correspondants une fois la pièce présentée. Les aides -on se prête la main d'un chantier à l'autre pour cette opération- poussent ou tirent de toutes leurs forces l'autre extrémité, jusqu'à coïncidence des deux bordages. Prestement, le chef de manœuvre enfonce alors toutes les gournables à grands coups de maillet, puis arase leurs têtes. Cette technique nécessite naturellement des bordés épais, de près de quarante mm. Cependant, ce n'est qu'au-delà qu'ils subissent un pré-cintrage, maintenus ployés dans un léger feu de palmes sèches (photo 2).

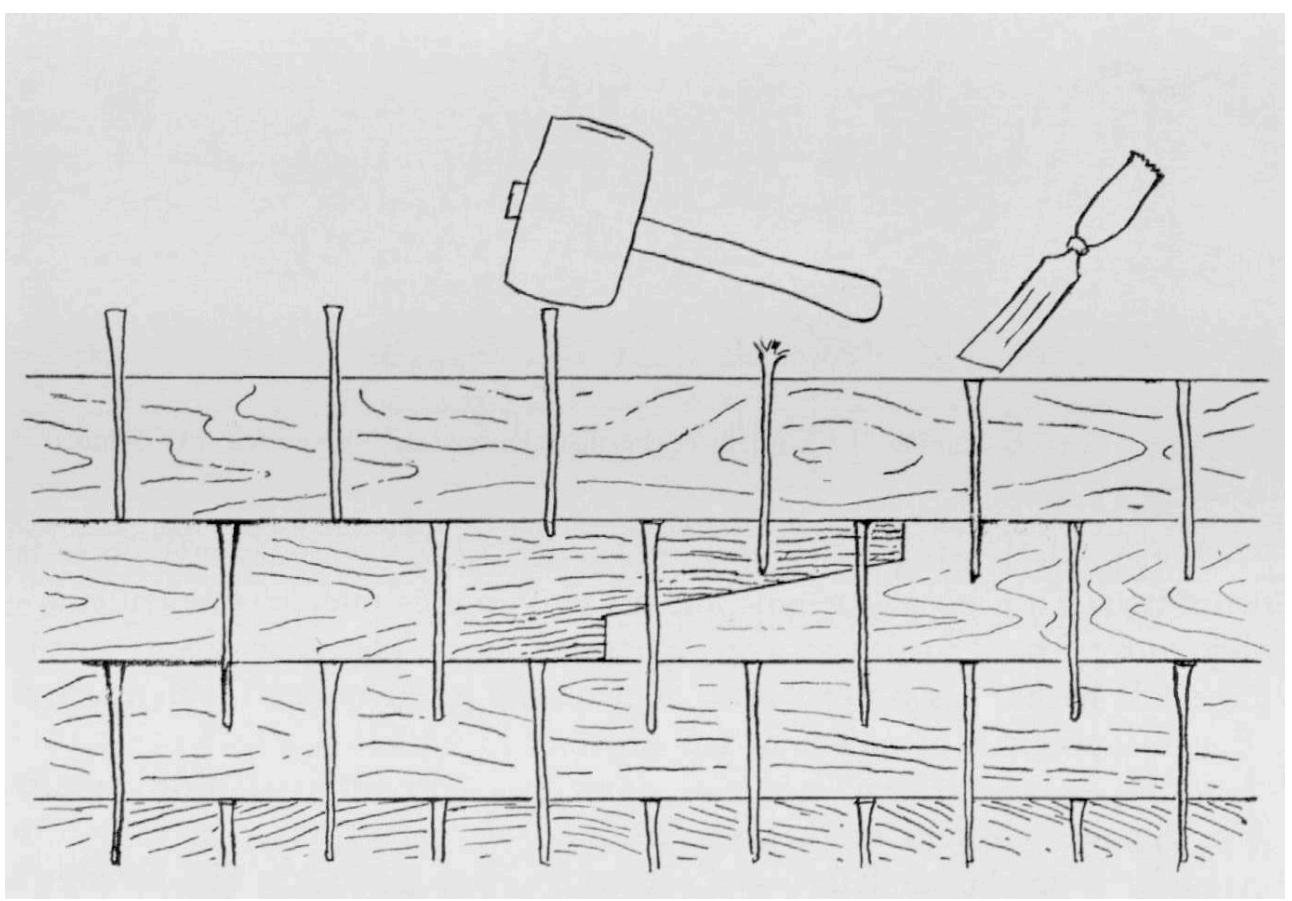

Figure 1. Chevillage par gournables en bois dur

Entre chaque virure est écrasée une mince lamelle fibreuse prélevée à l'intérieur de l'écorce du cocotier. Il n'y a pas de calfatage ultérieur, sauf magique - au lait maternel - mais cela est un autre sujet. Les coutures sont tout juste visibles. Le soin apporté à cette partie essentielle contraste avec le manque de finition du teste. Il faut aussi noter qu'on ne craint pas, comme en Chine, les écarts, réalisés en trait de Jupiter : on ne les double même pas de garnis. 


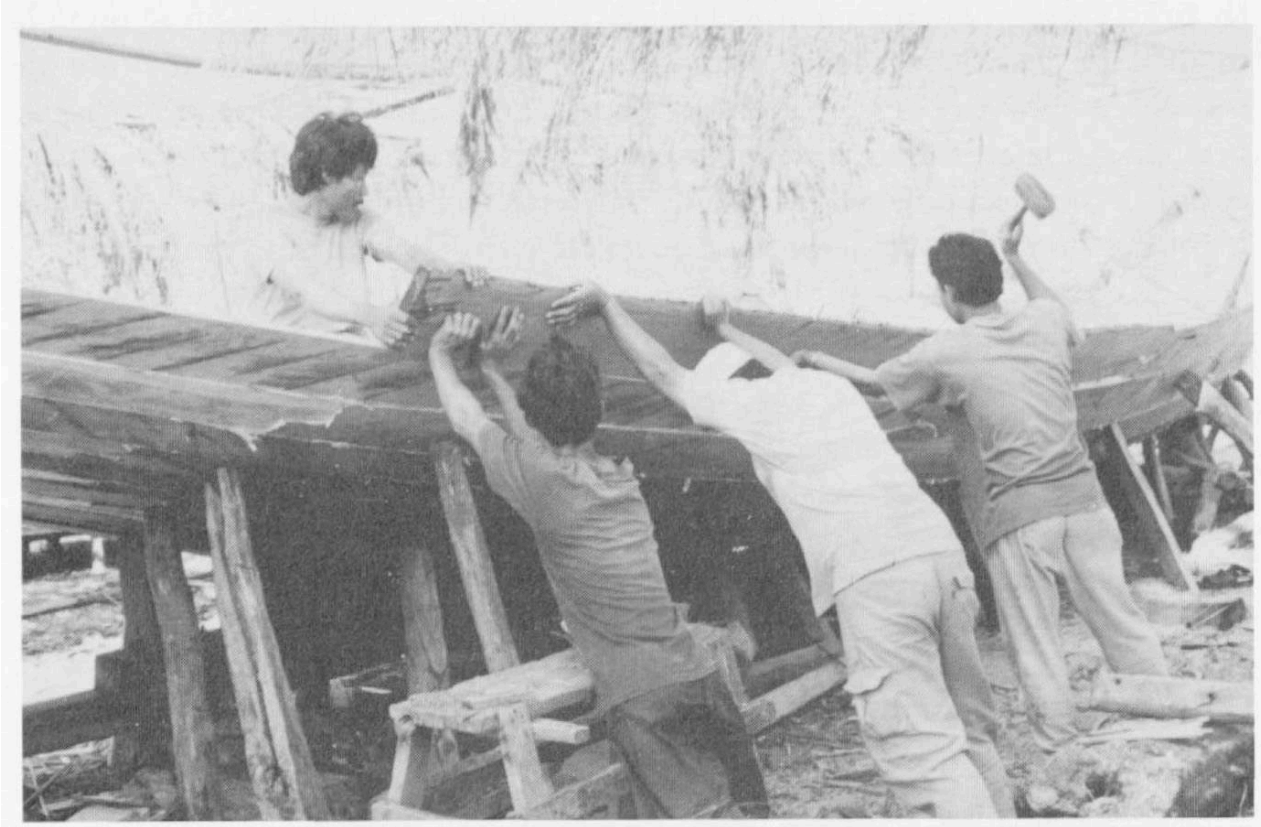

Photo 2. Cintrage manuel d'un bordage et chevillage en travers, Sulawesi (cl. J. Cl. Amos)

Lorsque le bordé parvient près du bouchain, on commence à poser la membrure. S'il n'y a pas de bois tors, de plus en plus rare, le charpentier applique un fer rond sur la concavité, le reporte sur un plateau et chantourne la pièce à la hache. Les couples sont alignés (sans recouvrement), varangues et allonges s'aboutant simplement par un trait de Jupiter. L'adhérence est recherchée, mais l'extérieur reste brut, ajoutant à cette impression de rugosité déjà signalée. Ce sont des bateaux de travail, et il faut serrer les coûts. En revanche la maille est faible, et l'échantillonnage généreux. Sur les unités destinées aux lourdes cargaisons on multiplie les serres. Le tout, naturellement, chevillé en bois (photo 3). Ni vis, ni clous. Tout au plus quelques boulons sur des pinisi. Il est difficile de décider si cette construction recherche la rigidité, comme en Occident, ou comme en Extrême-Orient, la souplesse.

Les unités construites dans la région ont un air de famille : élancements importants, étrave très apiquée, tonture accentuée, et, sauf le pinisi, fond plat. 


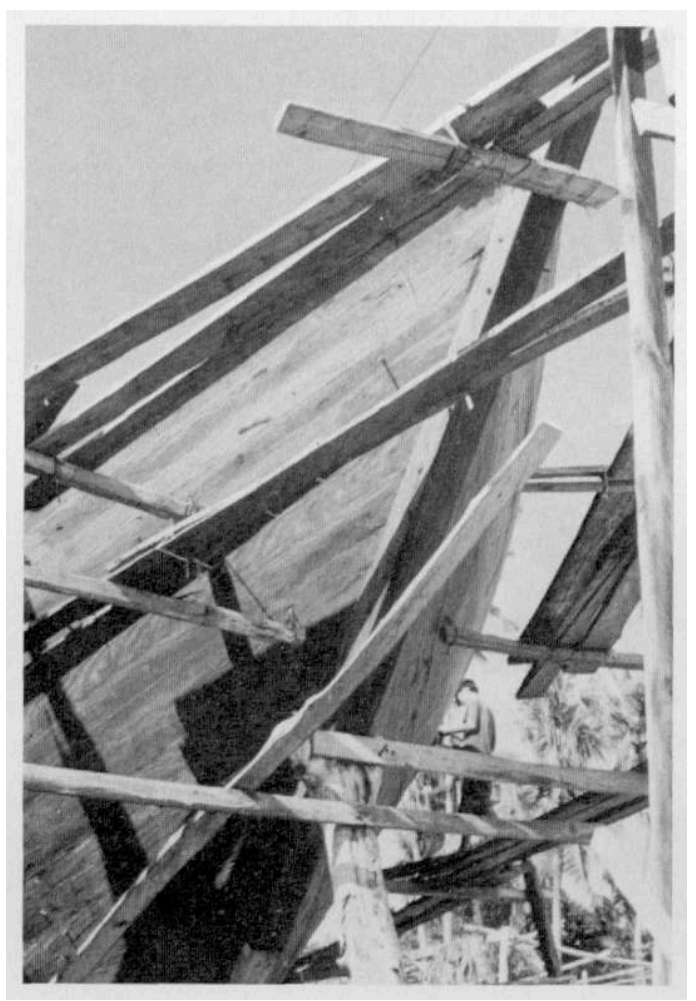

Photo 3. Pinisi motorisé, entièrement chevillé par gournables (cl. J. Cl. Amos)

\section{Pratiques magiques}

Traitant des bateaux bugis, comment ne pas évoquer les rites qui accompagnent leur création, nombreux, complexes, et toujours respectés? La croyance générale veut qu'une unité mal «baptisée» cherche à se mettre au plain. Le soupçon d'une négligence en la matière autorise l'armateur à suspendre la commande, et les travaux ne reprendront qu'après quelque exorcisme (ou lorsque les capitaux qui manquaient auront été réunis ?). On trouvera en annexe un témoignage de Pelly datant de 1977.

Les cérémonies décrites nous rappellent celles d'un chantier malais (khmer islam) du Cambodge, auxquelles nous participions en 1966. Dépôt votif encastré dans la quille (ici constitué des dons traditionnels du fiancé à sa promise ; y figure une pièce d'or comme celle que l'on plaçait en Europe sous le grand mât)... conjurations, prières (récitation de versets coraniques, en l'occurrence), sacrifices d'animaux, élaborations de talismans qu'on emportera en mer... Tout cela est universel et immémorial.

Chez les Bugis, l'accent est mis sur la «naissance » du bateau : termes sexuels appliqués aux mortaises et tenons des oeuvres maîtresses, aspersions, en guise de sang virginal, de celui d'un poulet, participation d'une femme enceinte aux cérémonies, et surtout, ce très curieux rite du percement, dans la quille, d'un «nombril », que l'on ne rebouchera qu'après le lancement. Le bébé humain va de l'eau à l'air, le bébé bateau de l'air à l'eau. Coupé chez l'un, le « cordon ombilical » est obturé chez l'autre (fig. 2). 


\section{Le pinisi nusantara (« pinasse de l'Archipel »)}

25 Le pinisi, orgueil des chantiers bugis, est aussi le voilier emblématique de l'Indonésie. Peu ancien, cependant, et n'ayant eu, en tant que voilier, qu'une durée de vie insignifiante, ce dundeene pouvait rivaliser en originalité avec ses concurrents commerciaux, les lété-létét de Madura, héritiers des prao mayang de Pâris, si surprenants avec leur pont au ras de l'eau et leur immense voile triangulaire océanienne.

26 Mais il a supplanté au milieu de ce siècle les autres voiliers de charge. Au début des années soixante-dix, le lambo, caboteur gréé en cotre franc, longtemps très actif, était en voie de disparition. Le lété-lété, généralement à quai dans une zone réservée, restait assez présent. Le pinisi prédominait largement et c'est la forêt de ses mâts que découvrait dans les ports, étonné par cette résurgence du passé, le voyageur occidental. Sa rencontre en mer faisait rêver.

Aujourd'hui motorisé, et disgracié par un château arrière de pétrolier, c'est toujours sous le nom de pinisi qu'il assure une notable partie du trafic entre les ports qui lui sont dévolus : Sunda Kelapa au nord de Jakarta, Paotere (jouxtant Ujung Pandang), Tanjung Perak de Surabaya, Bandjarmasin, et quelques autres, moins importants.

\section{Origines}

Les gros tonnages en Indonésie sont une tradition ancienne, signalée par des sources chinoises, qui évoquent des bateaux pouvant porter mille hommes... chiffre symbolique pour un chinois, bien entendu. Ce sont sûrement des unités importantes, proches des bateaux du Borobudur et des Corocore dessinés par Pâris, qui assurèrent la colonisation de Madagascar et autres lieux de l'Océan Indien à l'époque de Crîvijaya. Plus tard, les Portugais s'étonnèrent de rencontrer des vaisseaux plus importants que les leurs. 


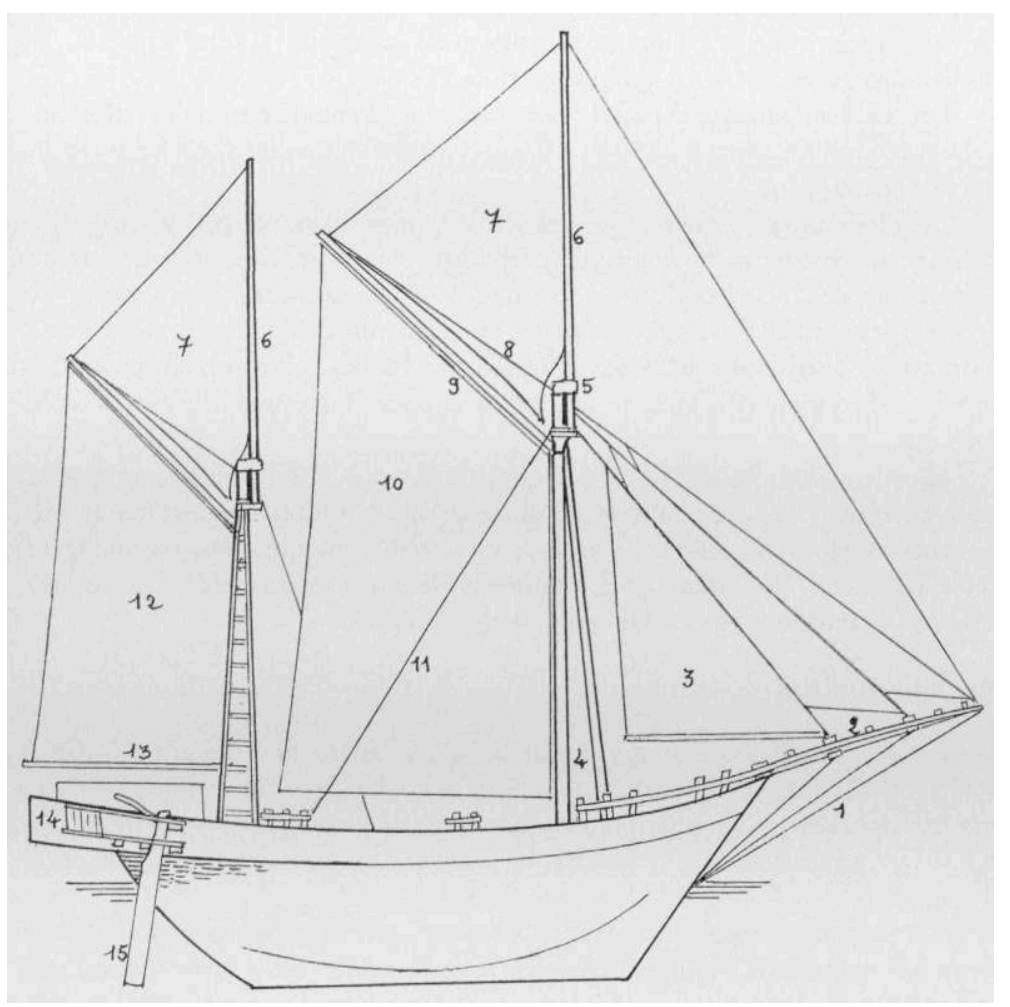

1 Sous-barbes (Pangango) 8 Martinet

2 Beaupré (Anjung) 9 Corne (Gak) (Bon atas)

3 Crinquette (Carengke) 10 Misaine (Layar utama)

4 Mât de misaine (Tiang agung) (l'échelle n'a pas été représentée) 11 Bastaque (Pondasi pengikat tali)

12 Artimon (L. belakang)

5 Chouquet 13 Bôme (Bon bawak)

6 Flèches 14 (Ambing)

Figure 2. Pinisi Nusantara

En 1767, le chevalier Pagès dessinait sur le vif une «trirème malaise " propulsée par deux immenses voiles carrées... et 184 rameurs. La taille des bateaux locaux avait d'ailleurs sensiblement diminué à cette époque, les Hollandais s'étant arrogé le gros du commerce ${ }^{5}$.

Les ancêtres directs du pinisi peuvent être cherchés parmi les caboteurs dessinés par Pâris entre 1829 et $1840^{6}$. Ces voiliers accusent une forte influence européenne.

Le pinisi aurait hérité son nom de la pinasse/pinacce par un singulier détour: le gréement de lougre de ce bateau d'Europe a été installé sur des barques locales, proches du pinisi tel que nous le connaissons. Il semble que ce gréement ait été ensuite remplacé par celui de goélette. On a vanté, et Conrad en particulier, les «schooners" de Macassar. Notons en passant la capacité d'adaptation des Indonésiens, tant pour le vocabulaire que pour les techniques.

C'est pendant la seconde guerre mondiale que le pinisi acquiert son aspect classique, en dépassant la capacité de cent tonnes de port en lourd: l'occupant japonais, faible en ressources navales marchandes, encourageait cette évolution. Désormais gréé en dundee, le pinisi règne sur la mer de Java, à peine concurrencé par les caboteurs de Madura. 


\section{Caractéristiques}

Nous décrirons d'abord le pinisi tel que nous l'avons observé entre 1968 et 1973 . Sa silhouette familière, sous voiles et au ras de l'eau, évoque la vieille marine occidentale, et plus précisément, les schooners américains de la côte Est. Mais, curieusement, les parties de cet ensemble ont toutes quelque chose d'original.

La voilure est impressionnante, la mâture plutôt grêle (photo 4). On se demande comment réagirait ce bateau dans la houle, mais la "méditerranée indonésienne » est un pot au noir ; quelques grains orageux surviennent, certes spectaculaires, mais avec plus de pluie que de vent et trop brefs pour faire lever la mer. C'est avant tout le manque de vent que craignent les équipages. On nous a pourtant affirmé à plusieurs reprises que des pinisi auraient assuré un trafic entre Jakarta et Vancouver. En attendant plus ample informé nous restons sceptiques...

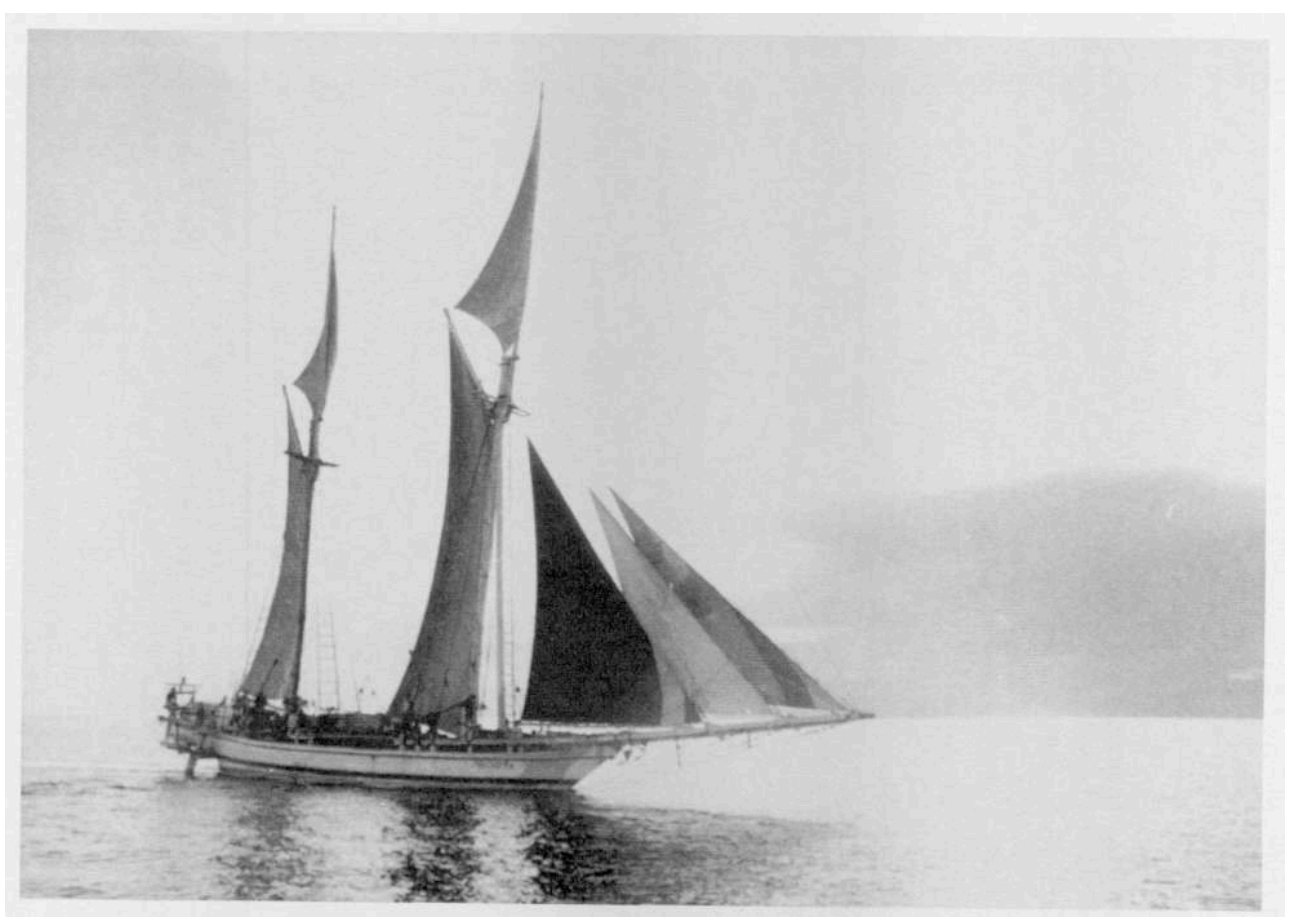

Photo 4. Un pinissi sous voile (1968, cl. J. Cl. Amos)

La coque, longue de 20 à $30 \mathrm{~m}$ (sans le beaupré) pour une largeur de 6 à $9 \mathrm{~m}$, a une maitresse section tonde, un bouchain obtus, des murailles très légèrement évasées, le maître bau sur l'avant. Rien que de très classique. On peut s'étonner de l'importance des élancements, surtout celui de l'avant, avec sa longue étrave rectiligne et singulièrement apiquée. Sans doute s'agit-il d'allonger le plan de voilure et, surtout, la flottaison. L'arrière est de type norvégien. D'épaisses préceintes font saillie sur la muraille. Les pavois se prolongent sur l'arrière pour encadrer une sorte de cage surplombante, surmontée d'une cabane ou d'une tente. Cet accastillage, d'architecture plus ou moins complexe, est un héritage du chebec arabe. De part et d'autre de ses ailes sortent les traversins auxquels s'amarrent les longs et lourds avirons de gouverne. L'ensemble est complété de sommaires «bouteilles». Le timonier, tourné vers l'avant, est juché sur une étroite et périlleuse plate-forme. Pour l'inciter à la vigilance, dit-on... Il gouverne sous le vent, mais les deux pelles sont manœuvrées de concert pendant les virements de bord. Le creux varie de deux mètres à plus de trois. Comme il n'y a ni 
faux-pont ni cloisons, cela donne des cales impressionnantes, entièrement dévolues à la marchandise... qui déborde d'ailleurs souvent sur le pont.

l'étarque, un hale-bas l'amène le long du mât, étou restant à poste. Le guindant est également endraillé (système rappelant ce qu'on nommait autrefois mât de corde). La bordure est libre, et les bastaques restent ridées au virement de bord. voile triangulaire, enverguée par un transfilage, et carguée en cas de besoin par un gabier grimpé dans la hune. La surface des voiles de flèche se justifie par la nécessité de "chercher l'air dans les hauts" (dans les accalmies, si fréquentes, une brise évanescente subsiste à partir de cinq à dix mètres de la surface).

On retrouve, grosso modo, les mêmes dispositions à l'arrière, mais l'artimon est généralement bômé (les bastaques étant ici moins vitales). Le mât est parfois unique (pour la même raison), et étayé. Il n'y a pas de rechanges pour les voiles, "tannées », noirâtres, indéfiniment rapiécées. La surface de voilure, sur un pinisi moyen, s'établit 
environ à $120 \mathrm{~m}^{2}$ de foc, autant pour la misaine, $90 \mathrm{~m}^{2}$ d'artimon, les voiles de flèche totalisant $70 \mathrm{~m}^{2}$. Soit $400 \mathrm{~m}^{2}$ en tout. C'est peu, bien que cette muraille de toile impressionne, et ces bateaux sont lents. Tout cela, cependant, peut être jugé par un homme du métier comme très marin.

En fait le pinisi, qui n'a guère évolué jusqu'à la motorisation, était dès ses débuts adapté à sa fonction, avec un minimum de complications et un coût très faible. On peut le considérer comme un exemple d'acculturation réussie.

\section{L'équipage}

L'équipage se compose de quinze à vingt hommes -plus qu'il n'en faut-, chacun affecté à un poste permanent. Les matelots dorment sur le pont. Il y a dualité de commandement. En mer le pilote est seul maître à bord. À terre le "capitaine " (capitaine d'armement, pourrait-on dire) reprend le pouvoir, s'occupe des formalités, $\mathrm{du}$ fret, décide de la prochaine escale. Tous, de préférence, appartiennent à la même communauté, macassar ou bugis.

Sur le pont se trouve un coffre à eau dans lequel nage un poisson, préposé au nettoyage, et la « cuisine », foyer rudimentaire. On mange du riz, un peu de poisson sec. Les prises en haute mer sont rares. On fume des kretek (cigarettes parfumées au clou de girofle)... quand il y en a. De temps à autre quelqu'un remue les pistons de la pompe de cale (deux tubes de bambou). En cas de mouillage forain, la pirogue posée sur le pont permet d'aller à l'aiguade, d'en rapporter quelques noix de coco.

Bien qu'il ne semble s'agir que de mers intérieures, les traversées sont longues. Près de huit cent milles à vol d'oiseau entre Jakarta et Ujung Pan-dang. Si le vent et le courant sont contraires, une errance misérable se poursuit pendant des semaines. En 1970, tien à bord ne ressemble à une carte ou un compas.

\section{Le pinisi actuel}

Les pinisi construits aujourd'hui à Maramusa et Tana Beru ont conservé leur beaupré, leur arrière de chebec. Une allonge de voûte, terminée par un tableau plat ou cintré, ne modifie pas essentiellement les lignes. Mais la poupe est écrasée par un énorme château, parfois surmonté d'une antenne parabolique. Du gréement subsiste un bipode, à l'avant, de la taille de l'ancien mât d'artimon. Peut-on parler de cargo mixte ? Ce modeste gréement est-il considéré comme un appoint, une sécurité, un hommage à la tradition... la justification d'une réduction des taxes?

Le moteur, abrégeant considérablement les traversées, a amélioré la vie des marins. De plus, ils disposent de couchettes, luxe impensable il y a trente ans. Stupeur! On a conservé les gouvernails latéraux ! Cette aberration résulterait, dit-on, d'une question de jauge fiscale (photo 5). Explication dont il faut tenir compre. De telles bizarreries ont été constatées ailleurs, notamment dans la Chine et le Viêt Nam d'autrefois. Mais on installe aussi un safran derrière l'hélice, bien entendu.

51 La préoccupation de l'esthétique, heureusement, subsiste en matière de peinture des coques. Les plus réussies, à notre goût, sont blanches, avec une bande bicolore épousant une virure au-dessus du bouchain. 


\begin{abstract}
La noria des portefaix -les mâts de charge restant exceptionnels- n'a pas changé. Nous avons vu un de ces hommes, d'apparence physique modeste, porter sur son dos au long d'une étroite planche une pile inquiétante de six sacs de farine : cent cinquante kilos au total.
\end{abstract}

encore. Mais le spectacle des ports réservés aux pinisi est toujours très coloré et animé.

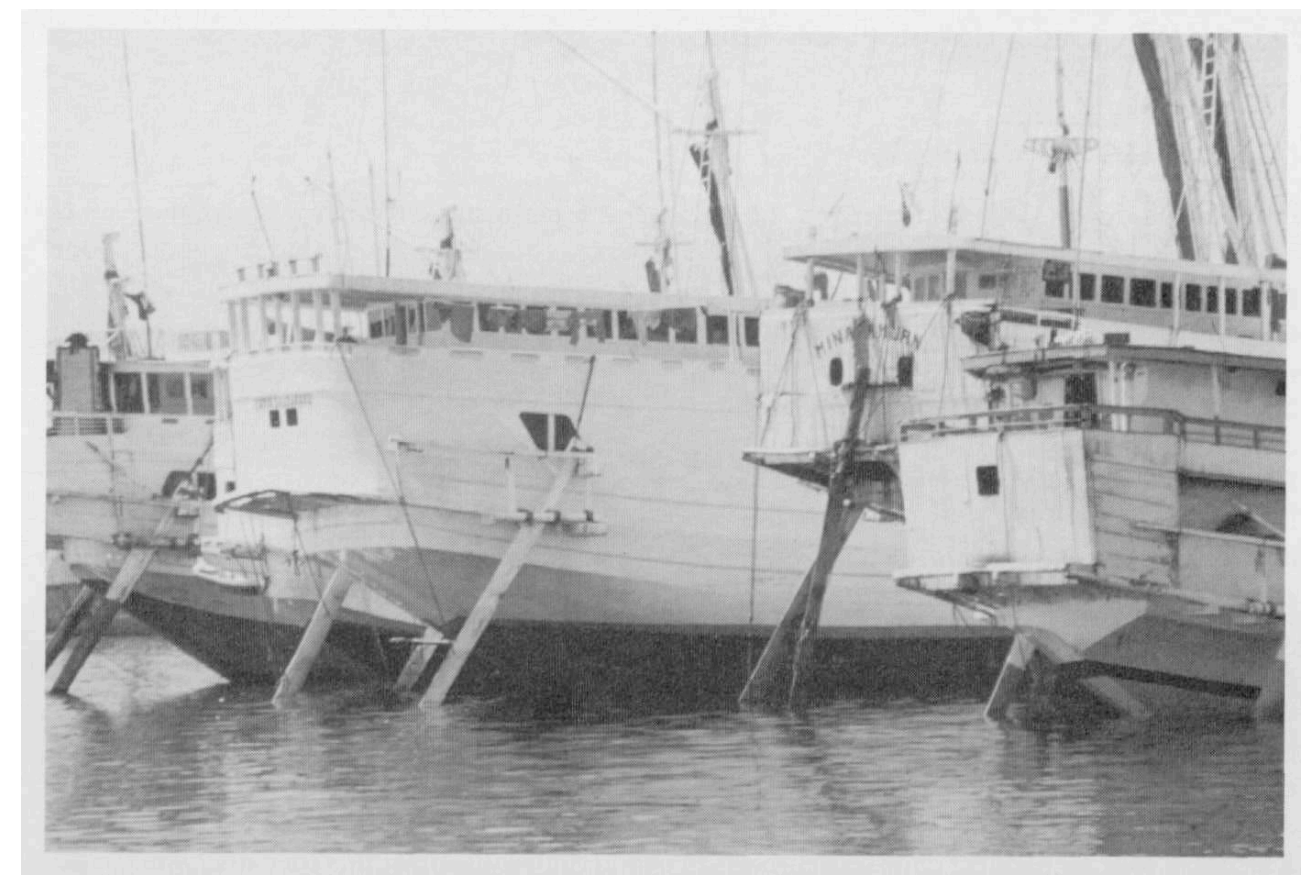

Photo 5. «Paotère » (Sulawesi). Ces pinissi motorisés ont conservé leur avirons de gouverne (juillet 1999, cl. J. Cl. Amos)

\title{
Le lancement
}

53 J'ai gardé pour la fin ce qui nous paraît le plus extraordinaire. La déclivité des plages est faible, le marnage se mesure en décimètres. Il faut, avec des moyens purement manuels, faire glisser quatre-vingt ou cent tonnes sur des rondins au long une centaine de mètres. Je n'ai pas assisté à la performance, mais, ayant été maitre d'oeuvre de la mise à l'eau, dans l'ouest de Java et selon les mêmes méthodes, d'un voilier de quinze tonnes, je peux me représenter l'opération, et admire qu'elle puisse être menée en un seul jour.

Les choses se passent ainsi. Toute la population mâle disponible -au moins deux cent personnes- est mobilisée. Deux équipes halent des haussières en cadence. Une autre, mains en l'air plaquées sous le bouchain, soulage la coque autant qu'il se peut. La dernière, plus réduite, déplace au fur et à mesure les accores de bambou qui préviennent le basculement. Le monstre, centimètre par centimètre, glisse vers l'eau où s'achèvera sa naissance. 


\section{Extraits de Symbolic Aspects of the Bugis Ship and Shipbuilding, par Usman Pelly (1977, traduction de J.- Cl. Amos).}

\section{Construction du bateau sur la plage}

Le point de départ de la construction du bateau sur la plage est le « premier assemblage de la quille " (en bugis, annatra kalebiseang, littéralement " couper quille»). Pour ce travail sont choisis un jour et une heure propices. C'est un fait traditionnel de la construction navale, qui se déroule dans une atmosphère sacralisée. Le jour prévu un madrier, qui deviendra la section centrale de la quille, est placé au centre du chantier. À cette cérémonie assistent le maître de hache, ses compagnons et apprentis, l'armateur et son épouse, le futur capitaine, et une femme enceinte.

Le maître est revêtu de blanc. Ayant récité une prière et allumé l'encens, il taille au ciseau l'extrémité de la quille préalablement choisie pour être à l'avant. En fait l'avant et l'arrière de toutes les pièces correspondent à leur position, au sommet ou au pied, dans l'arbre initial. Un copeau issu de ce premier travail est prélevé par le maître et coupé en deux moitiés, qui devront être conservées, l'une par lui, l'autre par l'armateur. Après quoi le maître poursuit son travail, dépouillé de son vêtement blanc. Des mortaises d'environ cinq centimètres de large sont creusées dans chaque extrémité de la partie centrale de la quille. On y introduira les tenons des deux autres sections, les lasso. Ces lasso sont les "raccords avant et arrière de la quille ». Avant d'insérer ces derniers on place dans les mortaises les objets suivants : une pièce d'or, du fer, du cuivre, du paddy, du riz brûlé croustillant, du riz cuit, du coco, et une feuille d'arbre, le tout empaqueté dans une cotonnade. Après quoi sont introduits les deux lasso.

Le maître de hache répand le sang de poulets, mâle et femelle, sur les assemblages. Les deux connections sont alors solidement ajustées. Puis il se lave la face avec de l'eau claire, marquant par ce geste que le rite est accompli. La femme de l'armateur s'approche, et lui fait un don (pasadagah), sous forme d'un anneau, de onze litres de riz blanc, trois sacs de paddy, et du vêtement blanc porté pendant la cérémonie. Enfin ils dégustent tous ensemble du wajik, gâteau de riz gluant.

La symbolique de ce rite est intéressante en plusieurs points. 1) Le partage du premier copeau, issu de la quille, entre le maître et l'armateur symbolise un accord, et même un lien entre les deux hommes. La moitié échue au second servira de talisman pour sauvegarder son bateau dans une éventuelle tempête, tandis que le premier pourra en user magiquement pour mener le bateau à sa perte si l'armateur l'a trompé (en ne réglant pas son dû ou toute autre vexation). 2) L'unité de la section centrale et des deux autres parties de la quille représente le «mariage» ou la «relation sexuelle». Les tenons s'appellent lasso, nom du pénis en bugis, et les mortaises sombong, celui du vagin. Le résultat de cette union sera un bébé : le bateau. 3) Bien plus, les objets placés dans les mortaises sont ceux que reçoit une jeune fille le jour de son mariage. Ils ont en euxmêmes une valeur symbolique : l'or signifie honneur et richesse, l'acier représente la force, le riz blanc la prospérité, et ainsi de suite. La présence d'une femme enceinte est le gage d'un fret abondant par la suite. Que ce «mariage » soit pur et sacré est mis en évidence par deux symboles: (a) le sang répandu sur la jonction des cléments de la 
quille, et (b) la qualité du bois utilisé pour la section centrale. Il ne peut être qu'excellent, jamais brûlé, foudroyé ou retiré de la mer. Ainsi est-il « vierge »...

\section{Cérémonies du lancement} Ce sont : (1) les chants islamiques (berzanji), (2) le façonnage d'un « nombril » du bateau
(pamossi), (3) l'offrande de nourriture aux habitants surnaturels (pakanre ballapatti) de la coque.

\section{Chants islamiques}

60 Les berzanji sont des hymnes à la gloire du prophète, habituellement chantés à l'occasion des circoncisions et cérémonies d'accueil des nouveaux mariés et nouveaunés. Ceci se passe au cours de la nuit précédant le lancement, en présence des notables de la communauté. En même temps se déroule le songkobala (invocations conjuratoires), mais les deux liturgies sont assurées par des personnes différentes. Le songkobala, de tradition bugis et préislamique, est ordonné par un panrita, spécialiste de ce rite. Tandis que le chœur populaire chante des cantiques, le panrita ne cesse de psalmodier des prières, ou mantra, tout en remuant dans un pot une décoction d'ingrédients magiques. Les chants ayant pris fin, les marins et le capitaine s'assemblent. Chacun est aspergé d'eau magique et en boit une gorgée.

\section{Création d'un « nombril » du bateau}

61 Cette action, faisant suite aux chants et aspersions, est assurée par le maître de hache dans la cale, en présence de l'armateur, du capitaine, des marins et des compagnons charpentiers. L'encens étant allumé, le maître se revêt de blanc et s'assied près du centre de la quille (kalebiseang). L'armateur, sa femme et le capitaine s'asseyent en face de lui, et les autres font le cercle. Une première chose est de déterminer le point ou se situera l'ombilic. L'ayant choisi, le maître le marque au ciseau. II détache un copeau et le place dans sa bouche avec l'anneau d'or reçu de la femme de l'armateur. C'est le riomong, ou «machouillage ». Il s'empare alors d'une tarière et, ayant récité une prière, fore la quille de bâbord vers tribord et de part en part, toujours revêtu de blanc. Cependant deux marins attendent sous la coque et recueillent les débris du perçage dans une poterie. On les placera dans une bouteille d'huile de coprah, de même que le copeau détaché au début du façonnage de la quille et conservé par l'armateur. Ayant foré le trou, le maitre se rince la bouche avec l'eau magique concoctée par le panrita et en crache un peu dans le «nombril ». L'armateur lui succède, et offre deux poulets, mâle et femelle. Leurs crêtes sont tranchées par le maître, et le sang coule dans l'ombilic. La cérémonie prend fin lorsque le maître de hache se lave la face. Chacun, alors, mange des gâteaux au riz gluant (wajik). Le percement du «nombril» est le dernier travail du constructeur et son ultime responsabilité. Plusieurs aspects symboliques de cette opération méritent d'être notés.

a) La réalisation du «nombril » est censée permettre d'augurer du sort du bateau. Si le trou débouche très légèrement au-dessus de l'angle inférieur tribord de la quille, la vie du bateau sera longue. Un peu trop haut, brève. Mais si la tarière sort sous la quille,

Techniques \& Culture, 35-36 | 2001 
alors le grand âge et la fortune du bateau sont assurés, tandis que la vie du maitre de hache sera tout au plus assez longue pour lui permettre de construite une nouvelle unité.

63 b) La bouteille contenant les reliefs du forage, le copeau initial et l'huile de coprah est suspendue au grand mât. Si le bateau, plus tard, est en proie à une fortune de mer, un peu de cette huile répandue près du « nombril » le sauvera du danger.

\section{Don de nourriture aux habitants surnaturels du bateau}

64 Cet épisode se situe à la suite de celui du «nombril», au milieu de la nuit. Seuls l'armateur, les marins et leur capitaine y assistent. L'offrande de nourriture aux génies est effectuée par un panrita, généralement proche parent du propriétaire du bateau. Le panrita est assis sur une estrade, face à l'ombilic, entouré par l'armateur et son épouse. Après avoir brûlé de l'encens, il récite une prière, et la femme lui donne un poussin, qu'il découpe de la tête à la queue ainsi qu'une fleur de bananier. Le tout est mélangé, et cette mixture placée dans de petits paquets que l'on disperse dans les parties essentielles, ombilic, extrémités de la quille, pied du mât...ce qui reste étant répandu autour du bateau. Cette cérémonie se poursuivra le lendemain, lorsque le bateau flottera sur l'onde. [N.B. L'auteur ne dit pas que pour les Bugis et d'autres peuples du sud-est de l'Asie les génies familiers des maisons et des bateaux se matérialisent sous l'apparence de lézards... d'autant plus utiles qu'ils pourchassent les cafards. On leur offre de la nourriture pour qu'ils daignent rester à bord. Celle qui est répandue sous la coque doit les inciter à faire revenir le bateau sur les lieux..., ].

Sous le centre de la quille est creusé dans le sable un trou d'un mètre carré, dans lequel on place une chèvre noire vivante. Recouverte de chutes de bois du chantier, elle est brûlée jusqu'à totale incinération. Cette cérémonie est un dernier sacrifice aux génies du chantier, peut-être une sorte d'action de grâces...

\section{Bateaux et humains}

Le peuple d'Ara (toponyme ancien?) crée un bateau comme une mère crée un bébé dans son ventre.

[...] Le lancement est appelé pasorong lopi (pousser dehors). Il représente la naissance. Tant que le bateau est en construction, un bon ordre doit être maintenu, aussi bien dans les relations entre travailleurs que dans celles du maître de hache avec l'armateur ou avec ses ouvriers. En ce qui concerne ces derniers une atmosphère détendue résulte certainement des liens familiaux. Mais chacun doit "se connaître lui-même " et se tenir à sa place. Le maître, en tant que chef, n'a pas à craindre de prendre des mesures disciplinaires, comme s'il s'agissait de ses propres enfants, s'il apparaît que quelqu'un se conduit de manière à troubler la bonne ambiance générale. Il peut s'agir de jeux d'argent, de bagarres, d'immoralités... Le maître s'efforce ainsi de créer une atmosphère d'ordre et d'harmonie.

Cette vision des choses est la même à l'égard d'une femme qui attend un enfant. L'équipe des travailleurs s'efforce de garder présent à l'esprit qu'une future mère a besoin de vivre dans une atmosphère d'ordre et d'harmonie, protégée de toute hostilité et adversité. Ils pensent qu'une mauvaise ambiance aurait de fâcheuses conséquences pour le bébé. 
69 choses sont liées, chacune n'étant qu'un aspect d'un grand Tout, la moindre infraction à la règle, que ce soit dans les techniques de construction ou dans les relations entre les constructeurs, aura des conséquences directes pour le bateau. Nous voyons donc pourquoi chacun s'efforce d'agir conformément à son rôle et à son statut. La plus grave infraction consiste en une offense de l'armateur à l'encontre du maître de hache, comme le défaut de paiement. En l'occurrence, selon la croyance générale, quant bien même le bateau serait terminé, son lancement sera difficile. Dans ce cas le maître n'y assiste pas. Si, passant outre, le bateau est lancé en force, on croit qu'il aura un destin sinistre, abîmé par la mer ou disparu sans espoir. De telle sorte que cette manière d'agir est assimilée au fait de forcer un bébé à sortir du ventre de sa mère : un avortement.

que les humains, les bateaux peuvent connaitre des périodes néfastes, comme s'ils étaient malades. Que font les Bugis sur un bateau en danger? La même chose que lorsqu'un enfant est sérieusement malade. Le père prendra un morceau du cordon ombilical de son enfant (coupé à la naissance, et suspendu à une poutre de la maison), le trempera dans l'eau chaude, et en frottera la tête, la poitrine et l'estomac de l'enfant. C'est ce que fait le capitaine dans un coup de vent. Il décroche la bouteille contenant les débris du «nombril » et suspendue au grand mât, et oint d'un peu d'huile le centre et l'avant de la quille, ainsi que quelques autres parties du bateau. En sorte que ce dernier, comme le bébé, échappe au danger.

\section{BIBLIOGRAPHIE}

Collins, G.E.P.

- 1937, Macassar sailing. Londres : Macmillan. Corre, Bruno

- 1987, Bornéo, Java, Bali. Paris : Sudestasie. Dick, H.W.

- 1975, « Perahu shipping in Eastern Indonesia », Bulletin of Indonesian Economic Studies XXI (2) :

95-114.

Hornell, James, 1936, Canoes of Oceania. Honolulu. Horridge, G. Adrian.

- 1979, The Konjo Boatbuilders and the Bugis Perahu of South Sulawesi. Maritime Monographs and Reports, National Maritime Museum, Greenwich.

Jannel, Claude et Frederic Lontcho, 1977, Le Dernier empire de la voile. Paris : Presses de la Cité.

Liebner, Horst, 1993, « Remarks on the terminology of boat building and seamanship in some languages of Southern Sulawesi », IC. 59/60:18-44.

Lineton, J., 1975, « Bugis migrants and wanderers », Archipel 10 : 173-201. Manguin, Pierre-Yves, 1980, « The South-East Asian ship : an historical approach », Journal of Southeast Asian Studies XI, 2.

Pâris, François-Edmond (Amiral), 1843, Essai sur la construction navale des peuples extra-européens, rééd. Grenoble, 1975. (Rééd. Fac-simile, Anthèse, Paris, 1990 ; rééd. partielle, E. Rieth. Voiliers et pirogues du monde au début du XIX ${ }^{\mathrm{e}}$ siècle. Ed. Du May, Paris, 1993).

Pelly, Usman, 1977, « Symbolyc aspects of the Bugis ship and shipbuilding », Journal of the Steward Anthropological Society 8 (2) : 87-106. Pelras, Christian

Techniques \& Culture, 35-36 | 2001 
- 1975, « Célèbes Sud fiche signalétique », Archipel 10 : 5-10 ; « La province de Célèbes Sud », Archipel $10: 11-50$.

\section{NOTES}

2. Au pluriel : pinisi-pinisi. Écrit jusqu'à une époque récente : pinisi2 (pratique officiellement abandonnée).

3. Nous traduisons ainsi punggawa, qui implique, en plus de la compétence, une autorité morale.

4. Ce nom étant incertain, nous nous proposions de mieux nous informer, mais, en 1999, ce bateau avait disparu.

5. Pour plus d'informations sur les origines, voir Manguin (1980).

6. Voir en particulier dans Pâris (1993) les planches 81 (toup de Malacca), 82 (lantcha). 84 (caboteurs malais), et, surtout, 96 (caboteurs de Macassar).

7. Voir photos dans Jannel et Lontcho (1977) et Corre (1987).

\section{RÉSUMÉS}

Il y a trente ans, les eaux de l'archipel indonésien étaient labourées de grands voiliers de haute mer évoquant tantôt la grande époque du trafic des épices, tantôt les bateaux de pierre du Borobudur. Il n'en reste plus un seul. Mais les chantiers qui les créèrent, s'adaptant peu ou prou à la motorisation, continuent à lancer des coques à l'ancienne de plus de cent tonneaux. Il y a lieu, avant que cette activité, condamnée à terme, ne perde ses caractères traditionnels, de préciser ou compléter les observations dont elle a déjà été l'objet.

Thirty years ago, the waters of the Indonesian Archipelago were furrowed with high sea sailing ships. They evoked one of the great periods of spice trading or the bas-relief stone boats of Borobudur. They have now all disappeared but the building site have, little by little, been adapted to the phenomenon of motorization and the building of hull continues in the ancient way that weight more than 100 tons. Before this activity is condemned to disappear and lose its traditional characteristics, it is important to give more precisions and complete the observation concerning them.

Hace treinta años, las aguas del archipiélago indonesio eran recorridos por grandes veleros de alta mar evocando unas veces la gran época del tráfico de las especias, otras veces los barcos de piedra de Borobudur. No queda ninguno. Pero los astilleros que los crearon se adaptan poco a poco a la motorización, y continúan a lanzar cascos como los antiguos de más de cien toneles. Sería necesario precisar o completar las observaciones de las cuales ha sido ya objeto esta actividad antes de que, condenada como está, no pierda sus características tradicionales.

\section{INDEX}

Mots-clés : Bugis, chantiers navals, construction navale, Indonésie, pinisi, Sulawesi 
AUTEUR

JEAN-CLAUDE AMOS

Professeur honoraire, 26400 Eygluy-Escoulin 